Network Working Group

Request for Comments: 1877

S. Cobb

Category: Informational

\title{
PPP Internet Protocol Control Protocol Extensions for Name Server Addresses
}

Status of this Memo

This memo provides information for the Internet community. This memo does not specify an Internet standard of any kind. Distribution of this memo is unlimited.

Abstract

The Point-to-Point Protocol (PPP) [1] provides a standard method for transporting multi-protocol datagrams over point-to-point links. PPP defines an extensible Link Control Protocol and a family of Network Control Protocols (NCPs) for establishing and configuring different network-layer protocols.

This document extends the NCP for establishing and configuring the Internet Protocol over PPP [2], defining the negotiation of primary and secondary Domain Name system (DNS) [3] and NetBIOS Name Server (NBNS) [4] addresses.

Table of Contents

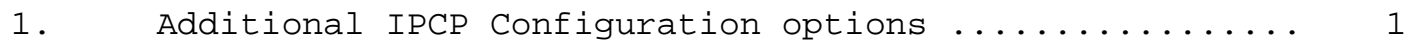

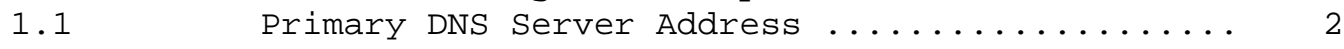

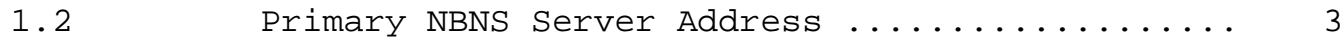

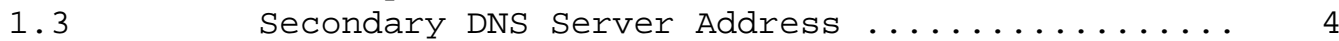

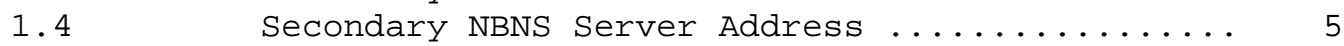

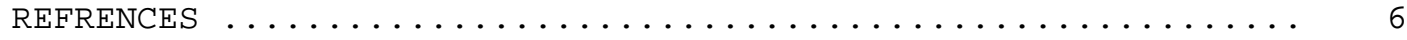

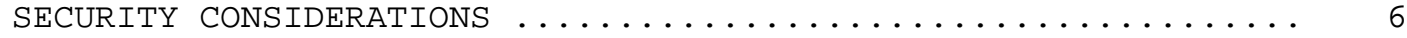

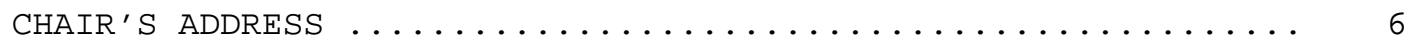

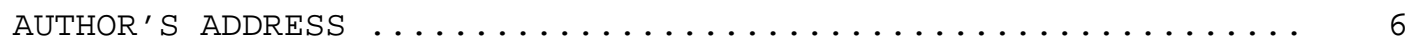

1. Additional IPCP Configuration Options

The four name server address configuration options, 129 to 132, provide a method of obtaining the addresses of Domain Name system (DNS) servers and (NetBIOS Name Server (NBNS) nodes on the remote network. 
Primary and secondary addresses are negotiated independently. They serve identical purposes, except that when both are present an attempt SHOULD be made to resolve names using the primary address before using the secondary address.

For implementational convenience, these options are designed to be identical in format and behavior to option 3 (IP-Address) which is already present in most IPCP implementations.

Since the usefulness of name server address information is dependent on the topology of the remote network and local peer's application, it is suggested that these options not be included in the list of "IPCP Recommended Options".

1.1. Primary DNS Server Address

Description

This Configuration Option defines a method for negotiating with the remote peer the address of the primary DNS server to be used on the local end of the link. If local peer requests an invalid server address (which it will typically do intentionally) the remote peer specifies the address by NAKing this option, and returning the IP address of a valid DNS server.

By default, no primary DNS address is provided.

A summary of the Primary DNS Address Configuration Option format is shown below. The fields are transmitted from left to right.

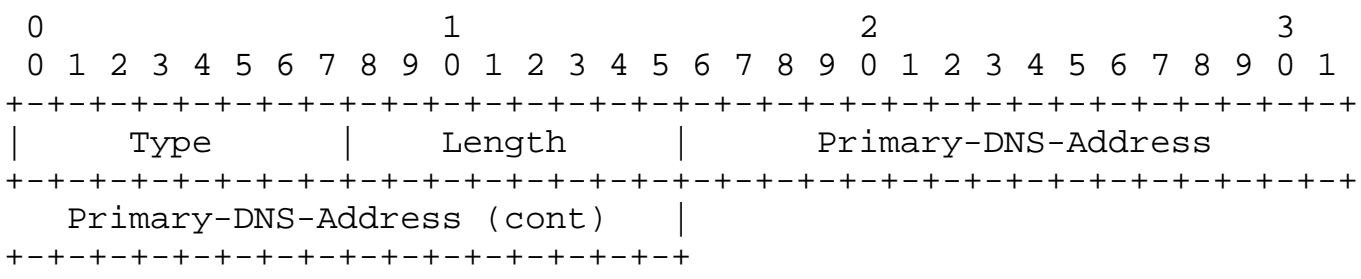

Type

129

Length 
Primary-DNS-Address

The four octet Primary-DNS-Address is the address of the primary DNS server to be used by the local peer. If all four octets are set to zero, it indicates an explicit request that the peer provide the address information in a Config-Nak packet.

Default

No address is provided.

1.2. Primary NBNS Server Address

Description

This Configuration Option defines a method for negotiating with the remote peer the address of the primary NBNS server to be used on the local end of the link. If local peer requests an invalid server address (which it will typically do intentionally) the remote peer specifies the address by NAKing this option, and returning the IP address of a valid NBNS server.

By default, no primary NBNS address is provided.

A summary of the Primary NBNS Address Configuration Option format is shown below. The fields are transmitted from left to right.

$\begin{array}{lllllllllll}0 & 1 & 2 & 3\end{array}$

$\begin{array}{llllllllllllllllllllllllllllllll}0 & 1 & 2 & 3 & 4 & 5 & 6 & 7 & 8 & 9 & 0 & 1 & 2 & 3 & 4 & 5 & 6 & 7 & 8 & 9 & 0 & 1 & 2 & 3 & 4 & 5 & 6 & 7 & 8 & 9 & 0 & 1\end{array}$

+-+-+-+-+-+-+-+-+-+-+-+-+-+-+-+-+-+-+-+-+-+-+-+-+-+-+-+-+-+-+-+-+

\begin{tabular}{l|l|l} 
Type & Length & Primary-NBNS-Address
\end{tabular}

$+-+-+-+-+-+-+-+-+-+-+-+-+-+-+-+-+-+-+-+-+-+-+-+-+-+-+-+-+-+-+-+-+$ Primary-NBNS-Address (cont)

$+-+-+-+-+-+-+-+-+-+-+-+-+-+-+-+-+$

Type

130

Length

6

Primary-NBNS-Address

The four octet Primary-NBNS-Address is the address of the primary NBNS server to be used by the local peer. If all four octets are set to zero, it indicates an explicit request that the peer 
provide the address information in a Config-Nak packet.

Default

No address is provided.

1.3. Secondary DNS Server Address

Description

This Configuration Option defines a method for negotiating with the remote peer the address of the secondary DNS server to be used on the local end of the link. If local peer requests an invalid server address (which it will typically do intentionally) the remote peer specifies the address by NAKing this option, and returning the IP address of a valid DNS server.

By default, no secondary DNS address is provided.

A summary of the Secondary DNS Address Configuration Option format is shown below. The fields are transmitted from left to right.

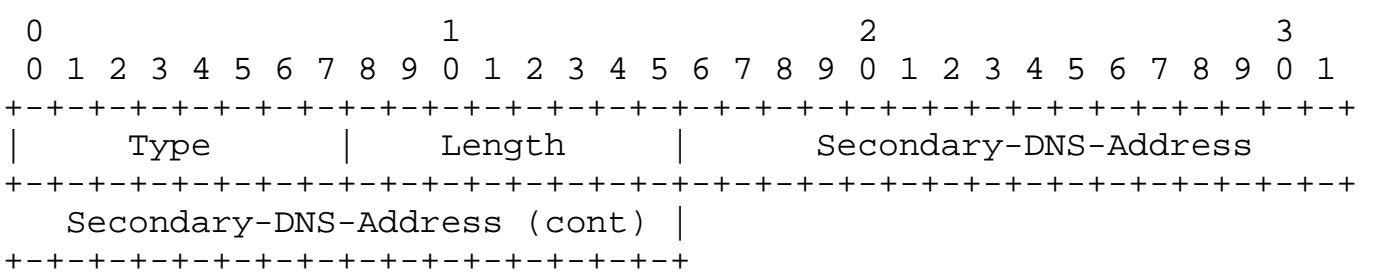

Type

131

Length

6

Secondary-DNS-Address

The four octet Secondary-DNS-Address is the address of the primary NBNS server to be used by the local peer. If all four octets are set to zero, it indicates an explicit request that the peer provide the address information in a Config-Nak packet.

Default

No address is provided. 


\subsection{Secondary NBNS Server Address}

Description

This Configuration Option defines a method for negotiating with the remote peer the address of the secondary NBNS server to be used on the local end of the link. If local peer requests an invalid server address (which it will typically do intentionally) the remote peer specifies the address by NAKing this option, and returning the IP address of a valid NBNS server.

By default, no secondary NBNS address is provided.

A summary of the Secondary NBNS Address Configuration Option format is shown below. The fields are transmitted from left to right.

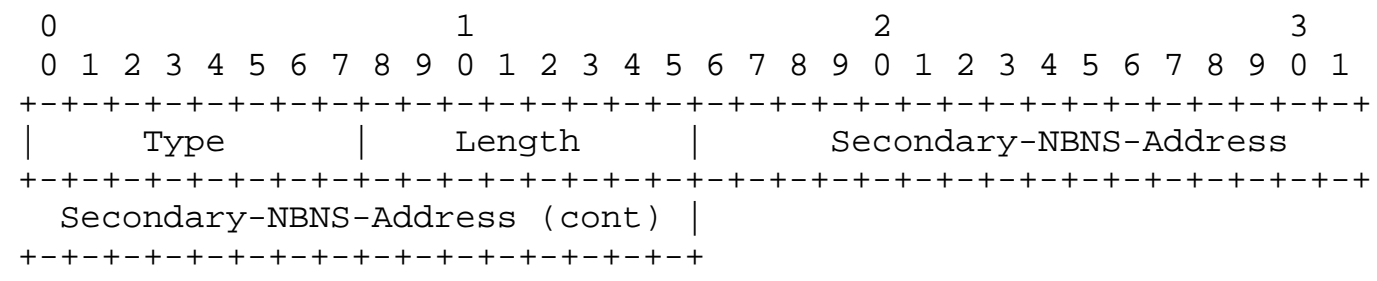

Type

132

Length

6

Secondary-NBNS-Address

The four octet Secondary-NBNS-Address is the address of the secondary NBNS server to be used by the local peer. If all four octets are set to zero, it indicates an explicit request that the peer provide the address information in a Config-Nak packet.

Default

No address is provided. 


\section{References}

[1] Simpson, W., Editor, "The Point-to-Point Protocol (PPP)", STD 51, RFC 1661, Daydreamer, July 1994.

[2] McGregor, G., "PPP Internet Control Protocol", RFC 1332, Merit, May 1992 .

[3] Auerbach, K., and A. Aggarwal, "Protocol Standard for a NetBIOS Service on a TCP/UDP Transport", STD 19, RFCs 1001 and 1002, March 1987.

[4] Mockapetris, P., "Domain Names - Concepts and Facilities", STD 13, RFC 1034, USC/Information Sciences Institute, November 1987.

[5] Mockapetris, P., "Domain Names - Implementation and Specification", STD 13, RFC 1035, USC/Information Sciences Institute, November 1987 .

Security Considerations

Security issues are not discussed in this memo.

Chair's Address

The working group can be contacted via the current chair:

Fred Baker

Cisco Systems

519 Lado Drive

Santa Barbara, California 93111

EMail: fred@cisco.com

Author's Address

Questions about this memo can also be directed to:

Steve Cobb

Microsoft Corporation

One Microsoft Way

Redmond, WA 98052-6399

Phone: (206) 882-8080

EMail: stevec@microsoft.com 